\title{
Maskless Direct Write Grayscale Lithography for MEMS Applications
}

\section{Curt McKenna, Kevin Walsh, Mark Crain, and Joseph Lake}

Dept. of Electrical and Computer Engineering, University of Louisville, Louisville, KY, USA

\begin{abstract}
Grayscale lithography is one area of lithography that has been relatively underutilized. There are several reasons for this, but one of the most prominent is the difficulty of modern techniques of grayscale exposure. However, this paper discusses a relatively novel approach to grayscale exposure using mask writing technology. Traditional lithography is characterized by the binary exposure of photoresist: meaning that some areas are exposed while other areas remain completely unexposed. The goal of grayscale lithography is to expose a gradient of intensities to photoresist. The result of this is a topography of photoresist that is potentially much more complicated than its binary counterpart.
\end{abstract}

This paper will discuss direct write grayscale lithography using the Heidelberg DWL 66FS Laser Pattern Generator. Traditionally the Heidelberg DWL 66FS is used for binary exposure, but it also has the ability to vary laser intensity during an exposure. By varying the intensity of the focused laser beam, the user is able to expose photoresist differently in various regions of the substrate, generating the grayscale structure.

\section{INTRODUCTION}

One of the most essential tools in microfabrication is photolithography, where a pattern is transferred onto a substrate by means of light exposure onto a photo-sensitive polymer. Until recently this process has been strictly binary, meaning that photoresist was to be either removed or entirely unaffected by the lithography process. However, new technologies in the lithographic field have led to grayscale lithography.

Grayscale lithography is a technique of exposure where various intensities of light are used to expose separate areas of a substrate differently. Using a relatively low intensity of light can mean that only a certain depth of photoresist is exposed, leaving the rest unchanged. The result from this can be the creation of a three dimensional structure in photoresist simply using lithographic techniques.

In this paper, we detail a method of direct write grayscale lithography using a Laser Pattern Generator. The writing method of the LPG will be explored along with an analysis of the resulting samples. Lastly, this paper will discuss the potential applications for this process and will note other uses for direct write grayscale lithography. [1]

\section{PROCESS}

This experiment was performed using a Heidelberg 66FS Laser Pattern Generator (LPG) at the University of Louisville Micro/Nanotechnology Center (see Fig 1). Traditionally the Heidelberg LPGs are suited only for binary exposures but the 66FS has the ability to vary laser intensity during a job process, thus lending itself towards grayscale lithography. It is by using this feature that we were able to generate the grayscale images.

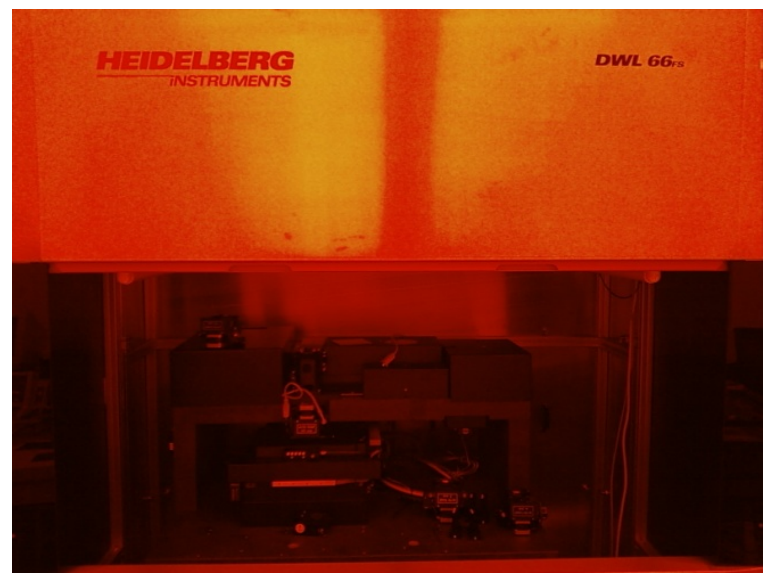

Figure 1 - University of Louisville Heidelberg 66FS Laser Pattern Generator

The process of creating a direct write grayscale lithography sample begins with creating the 
image in a layout editor such as L-Edit. The critical difference when designing grayscale layouts as compared with binary layouts is that each grayscale level must be defined as its own layer. The task of defining the light intensity for each of the layers can only be done once the file has been imported to the Heidelberg LPG. The light intensity and the type of photoresist used determine how deep an exposure will be. See figure 2 for an example of a grayscale layout.

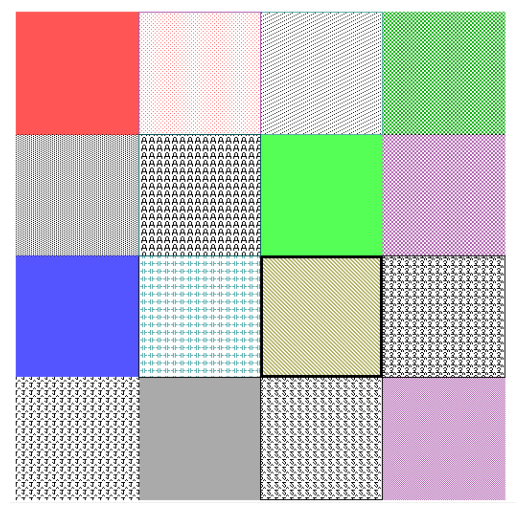

Figure 2 - L-Edit schematic of grayscale staircase using 16 distinct levels of exposure intensity

The photoresist plays a very important role in how the exposure is translated into an image. The photoresist that was used for these experiments was HD8820 Polyimide positive tone photoresist. This photoresist was spun onto a sample using a Headway spinner. We used a spread speed of 500 RPMs for 5 seconds and a spin speed of 4000 RPM for 30 seconds. Following this we performed a soft bake for 3 minutes at $125 \mathrm{C}$ on a hotplate at room temperature. We were able to obtain a photoresist thickness of roughly 5um on top of the sample. A thick photoresist is critical because various intensities of light will expose various depths of photoresist and if the photoresist is not thick enough it will result in different exposure levels both registering as fully exposed. [2]

The Heidelberg 66FS Laser Pattern Generator is very unique in the way it exposes photoresist. It works with a combination of linear passes along the length of the design along with a very fast sweeping motion by the laser. In this method it allows the Heidelberg LPG to quickly expose a larger area than other LPGs that lack the sweeping motion. This makes it much more practical for grayscale lithography, which requires more processing time than traditional binary exposures.

\section{The Heidelberg 66FS Laser Pattern} Generator is well suited to make a wide variety of structures based on its specifications. The minimum feature size in both the $\mathrm{x}$ and $\mathrm{y}$ directions are approximately $500 \mathrm{~nm}$, which allows for very resolute structures. University of Louisville's system is ideally suited for grayscale lithography because it has the only Heidelberg 66FS capable of 128 levels of laser intensity. This correlates into a potential of 128 unique levels present in photoresist after exposure. All other Heidelberg 66FS that are capable of grayscale lithography are only capable of 32 levels. [3]

Finally, a POLOS CPK wafer spinner was used to develop the sample (see Fig 3). This equipment was used for development because it has a very consistent and standardized spray method that allows it to consistently develop all parts of the sample evenly. Also, since it is programmable, it allows consistent results over the course of several samples. For these specific tests the following settings were used: 8 seconds of MF-319 spray, 10 seconds of MF-319 bath, 60 seconds of wafer rinse, and 45 seconds of wafer drying.

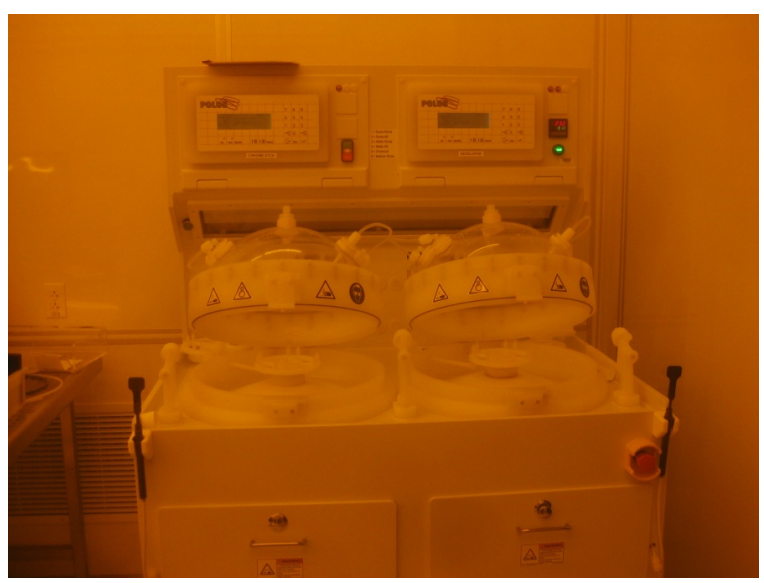

Figure 3 - University of Louisville POLOS CPK wafer spinner and spray developer 


\section{RESULTS}

Using this process the spiral staircase image shown in figures 4 and 5 was generated. This image features 16 distinct levels, each 250 um wide. For this image we designed a step height of slightly less than $10 \mathrm{~nm}$ to get a total image depth of about $150 \mathrm{~nm}$. The total area of the image is a $1 \mathrm{~mm}$ square. This design was created using only 16 layers in order to showcase the potential for drastic changes in exposures resulting in steps.

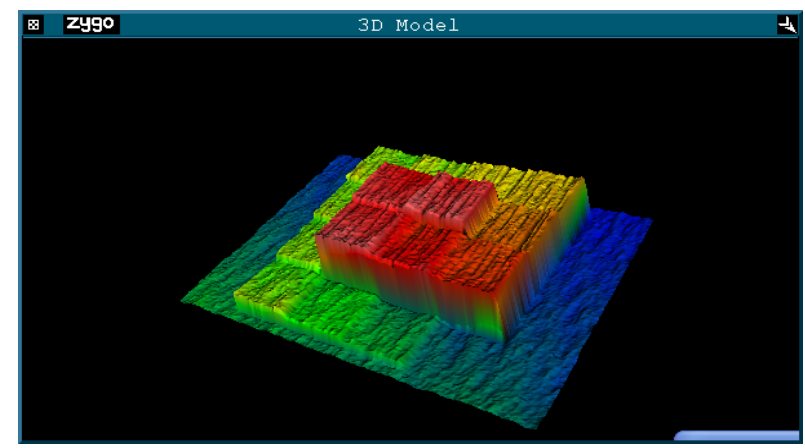

Figure 4 - Grayscale staircase in photosensitive polyimide featuring 16 levels of grayscale 3D model

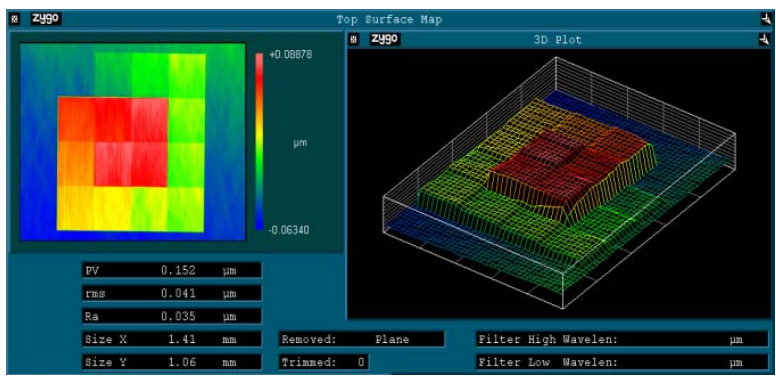

Figure 5 - Grayscale staircase in photosensitive polyimide featuring 16 levels of grayscale data plot

Another structure that we generated using grayscale lithography was a pyramid structure. Again this structure features distinct levels of exposure to produce steps. This structures, however, utilizes 32 levels of exposure. The resulting structure can be seen in Figures 6 and 7 . This structure features 32 pyramid steps with step sizes of roughly $70 \mathrm{~nm}$ resulting in a total structure height of $2.3 \mathrm{um}$. It is from this height that we can see the importance of using a photoresist of the proper thickness. Each step of the pyramid is 10um wide resulting in the base of the pyramid as a 640 um wide square. [4]

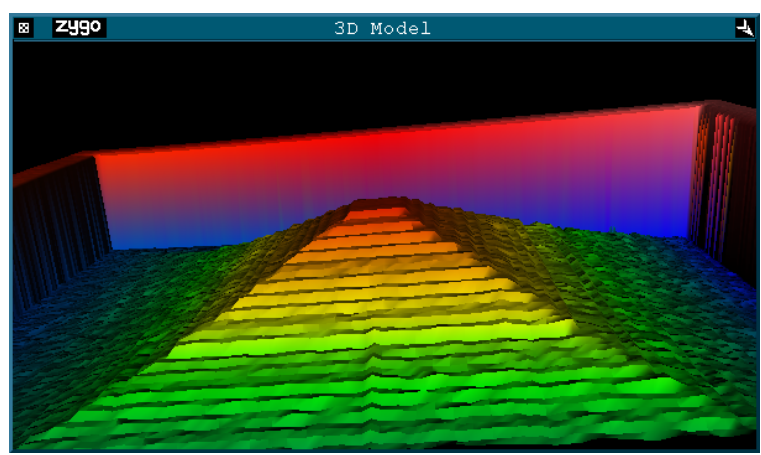

Figure 6 - Grayscale pyramid in photosensitive polyimide featuring 32 levels of grayscale 3D model

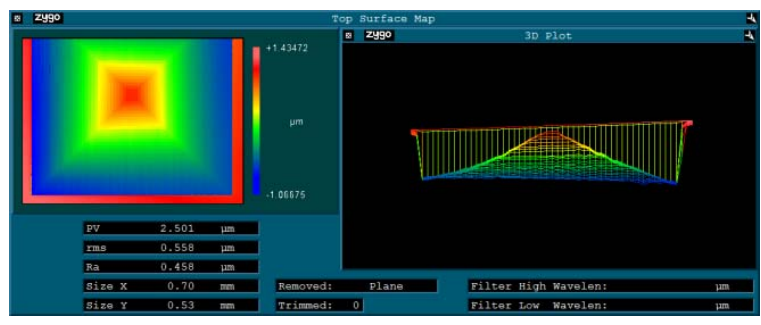

Figure 7 - Grayscale pyramid in photosensitive polyimide featuring 32 levels of grayscale data plot

This final 3D structure that we produced in resist was a replication of our Nanotechnology Building which features a sloped wall approaching a drop-off. This sloped structure was created by using 32 sequential exposure levels next to each other to create a smooth sloped surface. The width for each individual level was $3 \mathrm{um}$ and the width for the entire sloped structure was 96um. This experiment was performed to show the potential of creating sloped structures in photoresist using grayscale lithography.

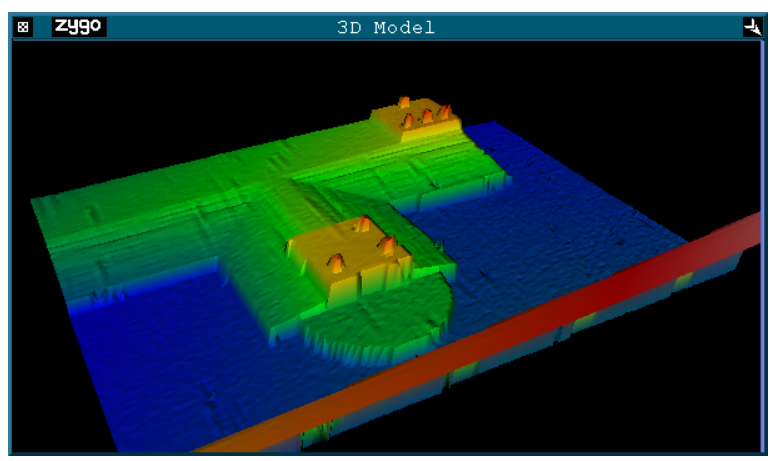

Figure 8 - Grayscale model of the UofL Nanotechnology Building produced in photosensitive polyimide 
These images were obtained using a Zygo New-View optical profiler. This equipment is advantageous because it does not apply force or heat to the sample to get the sample. For this reason there is no deformation of the photosensitive polyimide during observation.

\section{CONCLUSION}

This initial study of the Heidelberg 66FS has shown that it has great potential in the area of grayscale lithography. With 128 levels of grayscale and a higher resolution than most other grayscale options, the Heidelberg 66FS has the potential to make great strides in the field of grayscale lithography. The examples generated in this research showcase the potential for the Heidelberg.

One area of future research that can be explored is to use these grayscale exposed photoresists as masks to transfer images into a hard material such as silicon. This is planned to be done by using the Bosch process with Deep Reactive Ion Etching (DRIE). Since DRIE will etch through both silicon and polyimide, we can use this to etch the depth of the polyimide. This will cause the image that was only in polyimide to be transferred to the silicon. Using this hard 3D structure as a mold, it will then be possible to create multiple copies of a custom design using a subsequent process such as imprinting or embossing. With this process it will be possible to create complex structures much more easily than with other current technologies. [5]

\section{ACKNOWLEDGEMENTS}

This project was funded through a Navy contract entitled "MEMS Strategies for Critical Technology Protection" (Prime Contract \#DAAB0703-D-B010, Task Order 0198, Subcontract \#EGG0011378. This material is also based upon work supported by the National Science Foundation under Grant No. 0814194. Any opinions, findings, and conclusions or recommendations expressed in this material are those of the authors and do not necessarily reflect the views of the National Science Foundation.

\section{REFERENCES}

Guido Piaszenski, Ulrich Barth, Axel Rudzinski, Andreas Rampe, Andreas Fuchs, Markus Bender, Ulrich Plachetka, "3D structures for UV-NIL template fabrication with grayscale e-beam lithography", Microelectronic Engineering, Volume 84, Issues 5-8, Proceedings of the 32nd International Conference on Micro- and Nano-Engineering, May-August 2007, Pages 945-948

J.Kim; D.C. Joy; S.-Y. Lee; “Controlling resist thickness and etch depth for fabrication of 3D structures in electronbeam grayscale lithography," Microelectronic Engineering, Volume 84, Issue 12, December 2007, Pages 2859-2864

(3) "DWL66FS." Heidelberg Electronics Mikrotecnik GmbH. Heidelberg, July 2008. Web. 8 Mar 2010. $<$ http://www.himt.de/factsheets/dwl66fs.pdf $>$.

Lake, J.H.; Walsh, K.M.; McNamara, S.; , "Low-cost maskless grayscale lithography using a new photo-definable polyimide for polymer MEMS applications," Solid-State Sensors, Actuators and Microsystems Conference, 2009. TRANSDUCERS 2009. International, pp.1889-1891, 21-25 June 2009

Manish Kumar Hooda, Manoj Wadhwa, Sanjay Verma, M.M. Nayak, P.J. George, A.K. Paul, "A systematic study of DRIE process for high aspect ratio microstructuring", Vacuum, Volume 84, Issue 9, 19 April 2010, Pages 1142-1148, 